\title{
Linear fixed-field multipass arcs for recirculating linear accelerators
}

\author{
V. S. Morozov, ${ }^{1}$ S. A. Bogacz, ${ }^{1}$ Y. R. Roblin, ${ }^{1}$ and K. B. Beard ${ }^{2}$ \\ ${ }^{1}$ Thomas Jefferson National Accelerator Facility, Newport News, Virginia 23606, USA \\ ${ }^{2}$ Muons, Inc., Batavia, Illinois 60510, USA \\ (Received 6 April 2012; published 14 June 2012)
}

\begin{abstract}
Recirculating linear accelerators (RLA's) provide a compact and efficient way of accelerating particle beams to medium and high energies by reusing the same linac for multiple passes. In the conventional scheme, after each pass, the different energy beams coming out of the linac are separated and directed into appropriate arcs for recirculation, with each pass requiring a separate fixed-energy arc. In this paper we present a concept of an RLA return arc based on linear combined-function magnets, in which two and potentially more consecutive passes with very different energies are transported through the same string of magnets. By adjusting the dipole and quadrupole components of the constituting linear combined-function magnets, the arc is designed to be achromatic and to have zero initial and final reference orbit offsets for all transported beam energies. We demonstrate the concept by developing a design for a droplet-shaped return arc for a dogbone RLA capable of transporting two beam passes with momenta different by a factor of 2. We present the results of tracking simulations of the two passes and lay out the path to end-to-end design and simulation of a complete dogbone RLA.
\end{abstract}

DOI: 10.1103/PhysRevSTAB.15.060101

PACS numbers: 29.20.Ej, 29.27.Eg, 41.75.-i, 41.85.Ja

\section{INTRODUCTION}

Reusing the same linac in a recirculating linear accelerator (RLA) [1] for multiple beam passes provides for a more compact accelerator design and leads to significant cost savings. In a conventional RLA [2], the different-energy passes coming out of the linac are separated and directed into individual return arcs for recirculation. Thus, each pass through the linac requires a separate fixed-energy arc, increasing the complexity of the RLA. The maximum number of passes through the RLA's linac is often limited by design considerations for the switch-yard, which first spreads the different-energy passes to go into the appropriate arcs and then recombines them to align the beam with the linac axis. In this paper, we present a return-arc design concept, which allows two and potentially more consecutive passes with very different energies to be transported through the same string of magnets. In the proposed design, the arc is built of linear combined-functions magnets with variable dipole and quadrupole field components, which are adjusted to control the optics of the multiple passes.

The multipass arc design has a number of advantages over separate-arc [2] or pulsed-arc [3] approaches. It eliminates the need for a complicated switch-yard, it reduces the total beam-line length, there is no need to accommodate multiple beam lines in the same tunnel or construct separate tunnels for individual arcs, and there is no need for vertical bypasses, which may be required for separate arcs complicating the

Published by the American Physical Society under the terms of the Creative Commons Attribution 3.0 License. Further distribution of this work must maintain attribution to the author(s) and the published article's title, journal citation, and DOI. optics. This helps to increase the number of passes through the linac thus enhancing the top energy available with the same-size footprint. The design employs only fixed-field magnets, which alleviates the requirements on magnets and power supplies and greatly simplifies injection and extraction. The fixed-field design also allows for a rapid continuous-wave $(\mathrm{CW})$ acceleration. Another important feature of the design is a large dynamic aperture characteristic of linear-field lattices. It is fairly straightforward and inexpensive to design and build linear-field combinedfunction magnets even with relatively large apertures [4].

A dogbone-shaped RLA [5] with multipass arcs [6] was initially proposed for accelerating muons in the future Neutrino Factory and Muon Collider. Of all available particle species, accelerating a muon beam is, perhaps, the most challenging due to its large 6D emittance, short muon lifetime, and the wish to accelerate both muon charges in the same RLA simultaneously. Therefore, in this paper, we will be paying special attention to muon acceleration aspects. However, the multipass arc design concept is also applicable to both dogbone and racetrack RLA's for electrons and ions. Besides high-energy physics, such RLA's can find uses in free electron lasers and as accelerators for nuclear physics. Compact smaller-scale RLA's can benefit numerous applications [7-10] in industry, material science, astrophysics, medical isotope production, radiation cancer therapy, power generation, homeland security, and many other areas.

\section{DESIGN REQUIREMENTS}

Our goal is to configure the arc's magnetic structure so that it can accommodate multiple beam passes with substantially different energies. Keeping the emphasis on 
muon beams, the following constraints are imposed on the arc design.

(a) The arc's magnetic optics must be mirror symmetric for each pass, so that the beams of both charges can propagate through the arc in opposite directions equivalently. For a symmetric magnetic structure, this leads to the linear optics requirement that, for all passes, the Twiss $\beta$ functions must be equal and have equal-magnitude opposite-sign slopes (as a function of the longitudinal coordinate) at the beginning and end of the arc:

$$
\begin{gathered}
\beta_{x, y B}^{i}=\beta_{x, y E}^{i}, \\
\alpha_{x, y B}^{i}=-\alpha_{x, y E}^{i},
\end{gathered}
$$

where $\beta_{x, y}$ and $\alpha_{x, y}$ are the horizontal and vertical Twiss $\beta$ and $\alpha$ functions, respectively, subscripts $B$ and $E$ denote the locations at the beginning and end of the arc, and superscript $i$ refers to the pass number.

(b) Assuming a flat horizontal arc, each pass's reference orbit must have zero horizontal offset $x$ and zero slope $x^{\prime}$ (the prime denotes a derivative with respect to the longitudinal coordinate) at the beginning and end of the arc:

$$
\begin{aligned}
& x_{B}^{i}=x_{E}^{i}=0, \\
& x_{B}^{\prime i}=x_{E}^{\prime i}=0,
\end{aligned}
$$

to ensure that all energy beams are centered inside the linac.

(c) The horizontal dispersion $D_{x}$ and its slope $D_{x}^{\prime}$ must be zero at the beginning and end of the arc for all passes:

$$
\begin{aligned}
& D_{x B}^{i}=D_{x E}^{i}=0, \\
& D_{x B}^{\prime i}=D_{x E}^{\prime i}=0,
\end{aligned}
$$

to keep the linac dispersion free.

(d) The times of flight or, equivalently, the path lengths of the different-energy passes must provide proper synchronization with the linac.

(e) The orbit offsets as well as beta functions and dispersion must be maintained within reasonable limits for all energies to keep the magnet aperture sizes acceptable.

(f) The dynamic aperture and momentum acceptance at all energies must be adequate for large-emittance beams such as those of muons.

Since the arc's magnetic structure is mirror symmetric, if one chooses some values of $\beta_{x, y_{B}}^{i}$ and $\alpha_{x, y_{B}}^{i}$ and sets $x_{B}^{i}=0, x_{B}^{\prime i}=0, D_{x_{B}}^{i}=0$, and $D_{x_{B}}^{\prime i}=0$, it is sufficient to require that, at the arc's symmetry point,

$$
\begin{gathered}
\alpha_{x, y S}^{i}=0, \\
x_{S}^{\prime i}=0, \\
D_{x S}^{\prime i}=0,
\end{gathered}
$$

where subscript $S$ denotes the symmetry point location in the middle of the arc. The conditions given by Eqs. (7)-(9) impose a total of $4 \times i$ constraints. These constraints are optimal from the point of view of matching to the linac, however, they are not necessary requirements. We are intentionally considering a conservative case with the most constraints to illustrate the multipass arc concept. Depending on a specific situation, one might also consider relieving some of the constraints.

It is often convenient, especially for large arcs, to construct the lattice of identical superperiods. One must then apply the constraints of Eqs. (7)-(9) to each superperiod, i.e., indices $B, E$, and $S$ now refer to the beginning, end, and symmetry point, respectively, of the superperiod. In addition, to ensure proper matching between the superperiods, one must set $\alpha_{x, y B}^{i}=0$. This is equivalent to requiring that each superperiod has a periodic optics solution at each energy. Another advantage of this approach is that, under the discussed constraints, superperiods bending in opposite directions but otherwise identical are automatically matched to each other. This allows, for instance, construction of droplet-shaped return arcs, which include outward- and inward-bending sections. Reversing all bends in a superperiod preserves the linear optics but reflects the signs of $x$ and $D_{x}$. Given the constraints of Eqs. (3), (5), and (6), the opposite-bending periods are then still matched to each other.

Note that the points between the superperiods are convenient locations for insertion of any additional straight sections if needed because the dispersion there is suppressed for all passes and all-energy beams are centered in the beam line as per Eqs. (3), (5), and (6). Therefore, only $\beta$ function matching is required. Inserting samelength straight sections between all superperiods also preserves the arc's overall geometry.

\section{DESIGN APPROACH}

We first considered an arc design based on a nonlinear nonscaling fixed-field alternating-gradient (NS-FFAG) lattice [11]. The underlying structure was a triplet composed of in-out-in-(out-in-out-)bending combined-function magnets with fixed dipole and variable quadrupole, sextupole, and octupole field components. Having a few multipole components in each magnet provides a large number of knobs to satisfy conditions (7)-(9). An appropriate optics solution was demonstrated [6,12]; however, optimization of the nonlinear dynamics for multiple passes is rather challenging.

Therefore, we next investigated a linear NS-FFAG arc design. The lattice structure was similar to that of the nonlinear solution but each combined-function magnet only had fixed dipole and variable quadrupole field components. A solution based on the linear NS-FFAG approach was also demonstrated [13]. It had a number of advantages over the nonlinear design: (i) much greater dynamic aperture characteristic of linear-field lattices; (ii) simpler optimization of 
the nonlinear dynamics; (iii) simpler control of the reference orbit at each energy because of a lower sensitivity to magnetic field parameters than in the nonlinear case, which makes it easier to minimize the orbit excursion and adjust the path-length/time-of-flight; (iv) simpler control over the $\beta$ functions and dispersion; (v) lower sensitivity to magnetic field errors and magnet misalignments and simpler error correction; (vi) simpler combined-function magnet design with dipole and quadrupole field components only.

Since each combined-function magnet now only had one variable parameter, namely, the quadrupole strength, the number of magnets had to be increased to provide enough knobs to meet all of the constraints in Eqs. (7)-(9). Furthermore, because of the mirror symmetry, only onehalf of the magnets in a superperiod are independent. This made each superperiod longer and/or more finely structured. Another disadvantage shared by both the nonlinear and linear NS-FFAG-based designs is an inefficient use of the channel's length due to the alternating in-out-in (or outin-out) bends in the underlying triplet structure, i.e., bending the beam by a certain net angle requires a total bend of 3 times that angle. This makes the NS-FFAG-based designs relatively long and hard to compete with the separate-arc arrangement. In the concept presented in this paper, we deviate from the conventional FFAG scheme by not requiring regular alternating bends.

A solution satisfying the requirements discussed in Sec. II can be obtained using only same-direction bends, which would shorten the arc by almost a factor of 3 . However, there is still the problem of the large number of linear combined-function magnets required to satisfy Eqs. (7)-(9). Therefore, we introduce another concept [14] doubling the number of available parameters without increasing the number of magnets. We make the bending angles of some of the combined-function magnets variable with a constraint that the bending angles of all magnets in a superperiod must add up to a given fixed total bend:

$$
\sum_{n} \theta_{n}=\theta_{\text {net }}=\text { const, }
$$

where $\theta_{n}$ is the bending angle of $n$th magnet in the superperiod defined for a nominal-energy trajectory going along the center of the magnet and $\theta_{\text {net }}$ is the superperiod's total bending angle. Note that the superperiod's magnetic structure is maintained mirror symmetric, therefore, only a half of the magnets in the superperiod can have independent field parameters, namely, the bending angles and quadrupole strengths. Such an approach combines compactness of the design with all of the above advantages of a linear NS-FFAG scheme.

\section{LINEAR OPTICS}

We demonstrate the multipass arc concept in application to a proposed dogbone-shaped muon RLA [5] with two double-pass droplet return arcs. In that scheme illustrated in Fig. 1, both positively and negatively charged $0.9 \mathrm{GeV} / c$ muon beams are injected in the middle of a $0.6 \mathrm{GeV} /$ pass linac. The linac is then traversed by the beams 4 times accelerating them to $3.6 \mathrm{GeV} / c$. Therefore, one of the return arcs must accommodate 1.2 and $2.4 \mathrm{GeV} / c$ muon momenta, while the other arc must accommodate 1.8 and $3.0 \mathrm{GeV} / c$ momenta. Since the two arcs can be designed using the same approach, below we focus our discussion on the $1.2 / 2.4 \mathrm{GeV} / c$ arc whose design is somewhat more challenging due to the greater fractional momentum difference of the two passes.

Each droplet arc consists of a $60^{\circ}$ outward bend, a $300^{\circ}$ inward bend, and another $60^{\circ}$ outward bend so that the net bend is $180^{\circ}$. This arc geometry has the advantage that, if the outward and inward bends are composed of similar cells, the geometry automatically closes without the need for any additional straight sections, making it simpler and more compact.

To study the optics for large momentum offsets, we used the polymorphic tracking code (PTC) module [15] of MAD-X [16]. While perturbative-method codes are not suitable for such studies, PTC allows symplectic integration through all elements with user control over the precision with full or expanded Hamiltonian.

We assume that the arc is composed of identical superperiods. In our relatively low energy range, we use the maximum possible bend of $60^{\circ}$ per superperiod to have the largest possible number of magnets in the superperiod and, therefore, the largest number of free parameters for optics tuning. The superperiod consists of 24 combined-function magnets with dipole and quadrupole field components. For this initial demonstration, we chose hard-edge sector magnets because it is more straightforward to subdivide them into thin slices for tracking. The magnets are $0.5 \mathrm{~m}$ long and are separated by $0.2 \mathrm{~m}$ gaps. The total arclength is then $117.6 \mathrm{~m}$. Note that, in practice, it is more convenient to use

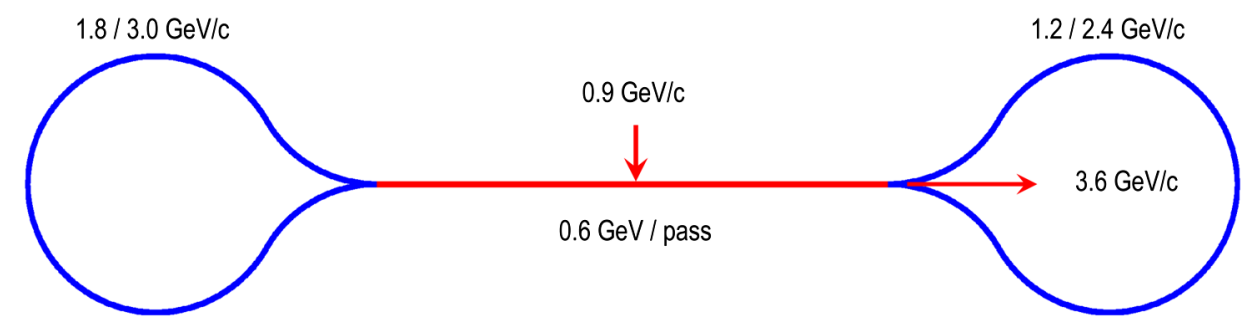

FIG. 1. Schematic layout of a 4.5-pass $3.6 \mathrm{GeV} / c$ muon RLA. 
rectangular magnets and there is no conceptual problem with converting the magnet shape in the solution from sector to rectangular. It is also straightforward to modify the design to include realistic magnet fringe fields.

Because of the droplet arc's geometric closing, the first and last few magnets of the arc overlap. These magnets cannot contain quadrupole field components because the symmetry would otherwise lead to the quadrupole fields having opposite slopes in the overlapping magnets. Therefore, we keep the first two magnets of each superperiod as pure dipoles. Their bending angles are fixed and are chosen to provide a sufficient separation of the incoming and outgoing higher-momentum beam while keeping the separation of the lower- and higher-momentum beams within acceptable limits. Also based on these considerations, we choose the higher $2.4 \mathrm{GeV} / c$ momentum as the reference momentum going through the magnet centers.

The beam trajectories at the beginning of the arc are shown in Fig. 2. After the first two magnets, the distance of about $33 \mathrm{~cm}$ between the incoming and outgoing $2.4 \mathrm{GeV} / c$ orbits is perhaps enough to insert separate magnets in the incoming and outgoing lines. For simplicity and to illustrate our multipass concept more clearly, the design presented in this paper is composed of identical symmetric supercells, which means that there are pure dipoles at the beginning and end of each supercell. However, none of the arc's inner magnets have to be pure dipoles. This opens another avenue for design optimization, in particular, one can reduce the orbit excursion and therefore the required magnet apertures inside the arc.

Note that a racetrack geometry does not have the above pure-dipole spreader/recombiner restriction, i.e., all magnets in a racetrack arc can have both dipole and quadrupole field components, which can help minimize the orbit excursion everywhere in the arc. Additionally, neither geometry requires that one of the passes has to go through the magnet centers. A solution can be obtained when momenta of all passes are different from the nominal center-line momentum [13]. This might be particularly useful for adjusting the path-lengths/times-of-flight of the different passes for synchronization with the linac.

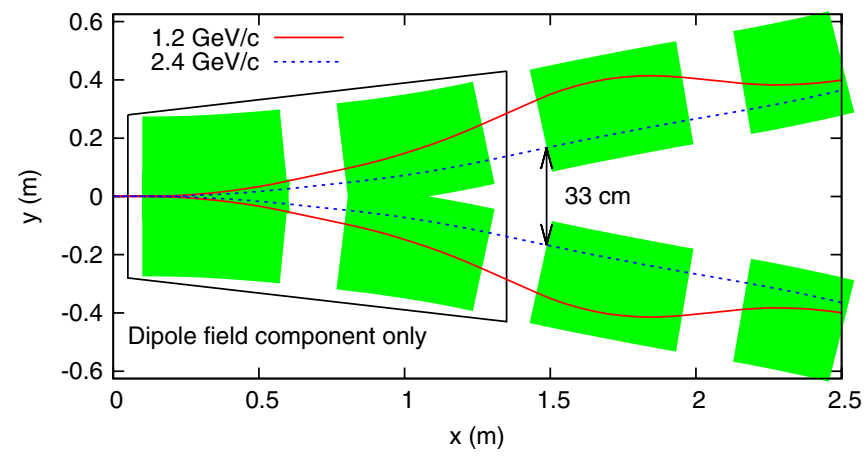

FIG. 2. The apex of the droplet arc with the pure dipoles acting as a spreader/recombiner of the different-momentum trajectories.
As discussed in Sec. II, each superperiod is symmetric with respect to its center. Therefore, out of the 24 magnets constituting the superperiod, 12 are independent. The first two of these magnets are pure dipoles with fixed bending angles of $6^{\circ}$ each. The remaining 10 magnets each have variable dipole and quadrupole field components with a constraint of Eq. (10) on the bending angles that the superperiod's net bend is $60^{\circ}$. This gives a total of 19 independent parameters.

Following the description in Sec. II, when solving for the 1.2 and $2.4 \mathrm{GeV} / c$ reference orbits and optics of the superperiod, the beginning values of the orbit offset $\left(x_{B}^{i}\right)$, dispersion $\left(D_{x B}^{i}\right)$, their slopes $\left(x_{B}^{\prime i}\right.$ and $\left.D_{x B}^{\prime i}\right)$, and the $\alpha$ functions $\left(\alpha_{x, y B}^{i}\right)$ were all set to zero for both momenta. The initial values of the horizontal and vertical $\beta$ functions $\left(\beta_{x, y B}^{i}\right)$ were all set at $2 \mathrm{~m}$ for both momenta to provide easy matching to the linac and to keep the peak values of the $\beta$ functions in the superperiod at acceptable levels. The 19 independent magnet parameters discussed above were then tuned to meet the requirements of Eqs. (7)-(9), i.e., to give zero slopes of the orbit offset, dispersion, and $\beta$ functions at the center of the superperiod for the two momenta. The symmetry then ensures that Eqs. (3)-(6) are satisfied at the superperiod's exit.

Since the $2.4 \mathrm{GeV} / c$ beam goes through the magnet centers, its reference orbit by definition has zero offset everywhere. This results in a total of 2 passes $\times$ 4 constraints/pass $-1=7$ constraints with 19 fitting parameters available. The extra free parameters were used to control the maximum values of the orbit deviation, $\beta$ functions, and dispersion. The resulting magnet parameters in the first half of the outward-bending superperiod are listed in Table I. In terms of magnetic field requirements, the maximum dipole field in Table I is about $1.7 \mathrm{~T}$ while the maximum quadrupole gradient is about $28 \mathrm{~T} / \mathrm{m}$.

TABLE I. Bending angles $\theta$, bending radii $\rho$, dipole fields $B$, and quadrupole strengths $\partial B_{y} / \partial x$ of the magnets in the first half of the outward-bending superperiod. The parameters are defined with respect to the channel's nominal axis coinciding with the $2.4 \mathrm{GeV} / c$ reference orbit.

\begin{tabular}{lrrrr}
\hline \hline Magnet \# & \multicolumn{1}{c}{$\theta\left(^{\circ}\right)$} & $\rho(\mathrm{m})$ & \multicolumn{1}{c}{$B(\mathrm{~T})$} & $\partial B_{y} / \partial x(\mathrm{~T} / \mathrm{m})$ \\
\hline 1 & -6.000 & 4.775 & -1.677 & 0.000 \\
2 & -6.000 & 4.775 & -1.677 & 0.000 \\
3 & 1.976 & 14.497 & 0.552 & 19.529 \\
4 & -5.008 & 5.720 & -1.399 & -24.584 \\
5 & -2.823 & 10.149 & -0.789 & 28.342 \\
6 & -2.572 & 11.140 & -0.719 & -22.321 \\
7 & 0.501 & 57.222 & 0.140 & 21.206 \\
8 & -1.950 & 14.691 & -0.545 & -21.230 \\
9 & -1.763 & 16.245 & -0.493 & 23.233 \\
10 & -2.472 & 11.588 & -0.691 & -27.653 \\
11 & -1.982 & 14.456 & -0.554 & 24.536 \\
12 & -1.907 & 15.023 & -0.533 & -18.612 \\
\hline \hline
\end{tabular}




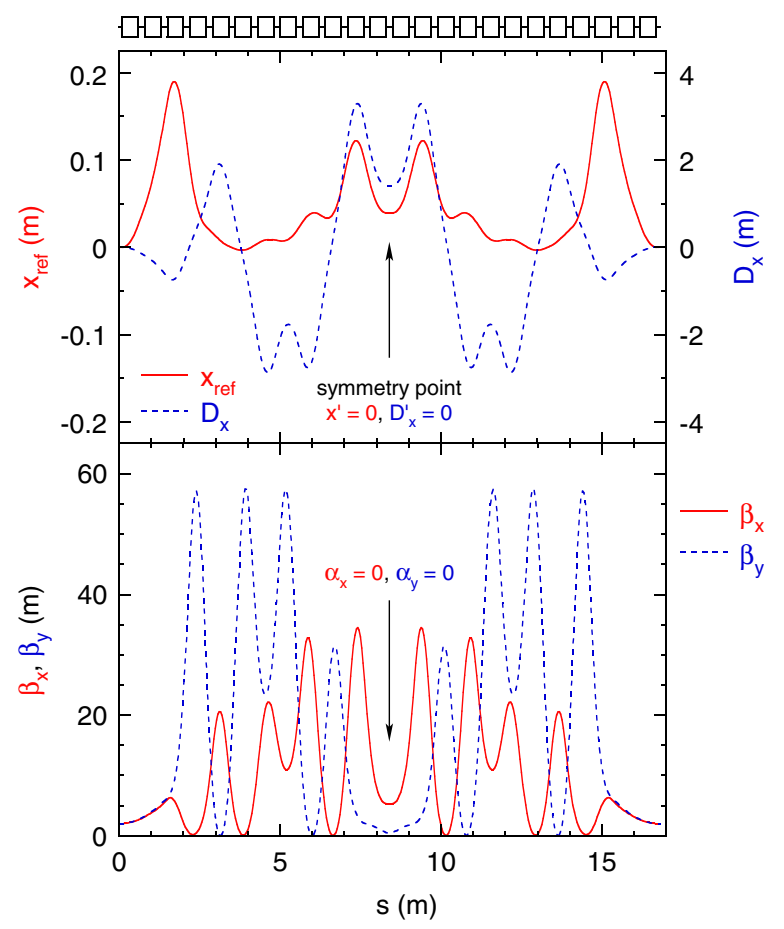

FIG. 3. $1.2 \mathrm{GeV} / c$ optics of an outward-bending superperiod: the horizontal reference orbit $x_{\text {ref }}$ and dispersion $D_{x}$ (top) and the horizontal and vertical $\beta$ functions (bottom).

Figures 3 and 4 show 1.2 and $2.4 \mathrm{GeV} / c$ solutions, respectively, for the periodic orbits, dispersion, and $\beta$ functions of an outward-bending superperiod. Since the only difference of an inward-bending superperiod from the outward-bending one is that its bends are reversed, its

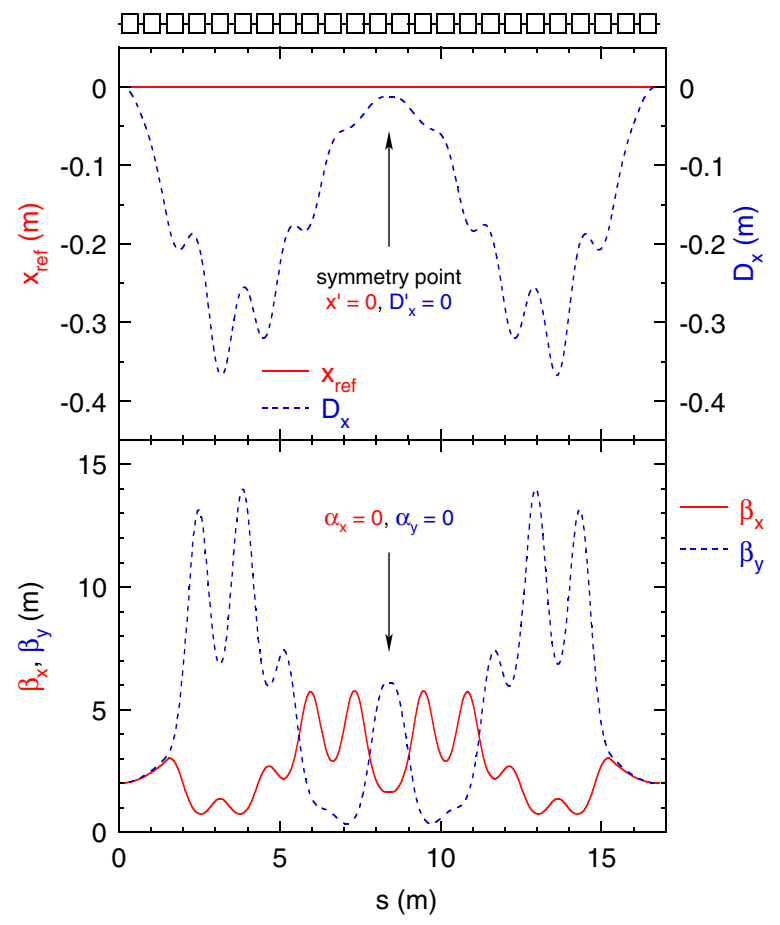

FIG. 4. $2.4 \mathrm{GeV} / c$ optics of an outward-bending superperiod: the horizontal reference orbit $x_{\text {ref }}$ and dispersion $D_{x}$ (top) and the horizontal and vertical $\beta$ functions (bottom).

optics is identical to Figs. 3 and 4 except that the signs of the reference orbits and dispersion are flipped. The $1.2 \mathrm{GeV} / c$ optics of a complete droplet arc is shown in Fig. 5. It illustrates how the whole arc is built out of individual outward- and inward-bending superperiods.

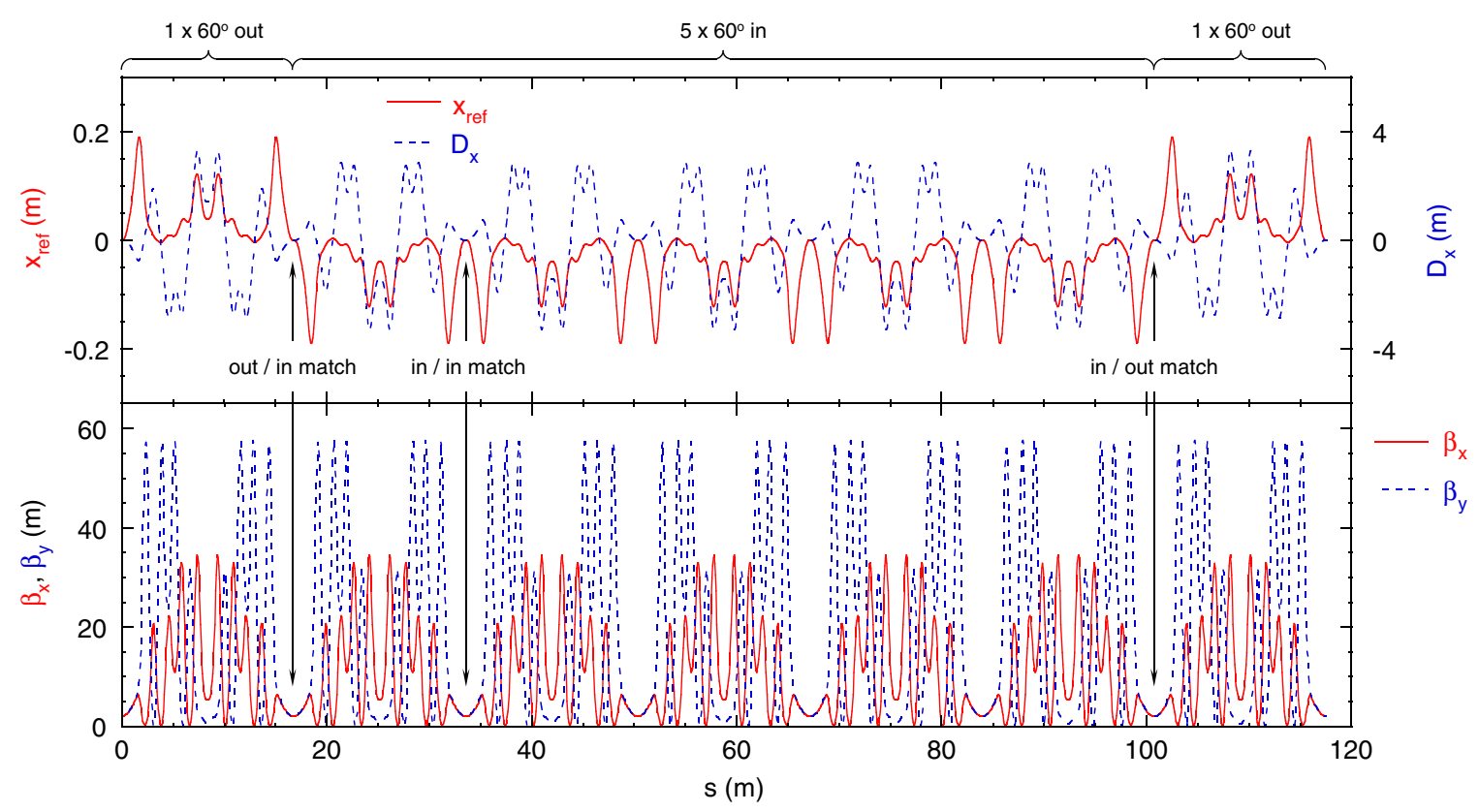

FIG. 5. $1.2 \mathrm{GeV} / c$ optics of a complete droplet arc: the horizontal reference orbit $x_{\text {ref }}$ and dispersion $D_{x}$ (top) and the horizontal and vertical $\beta$ functions (bottom). The arrows point to the different-type junctions between the outward- and inward-bending superperiods. 


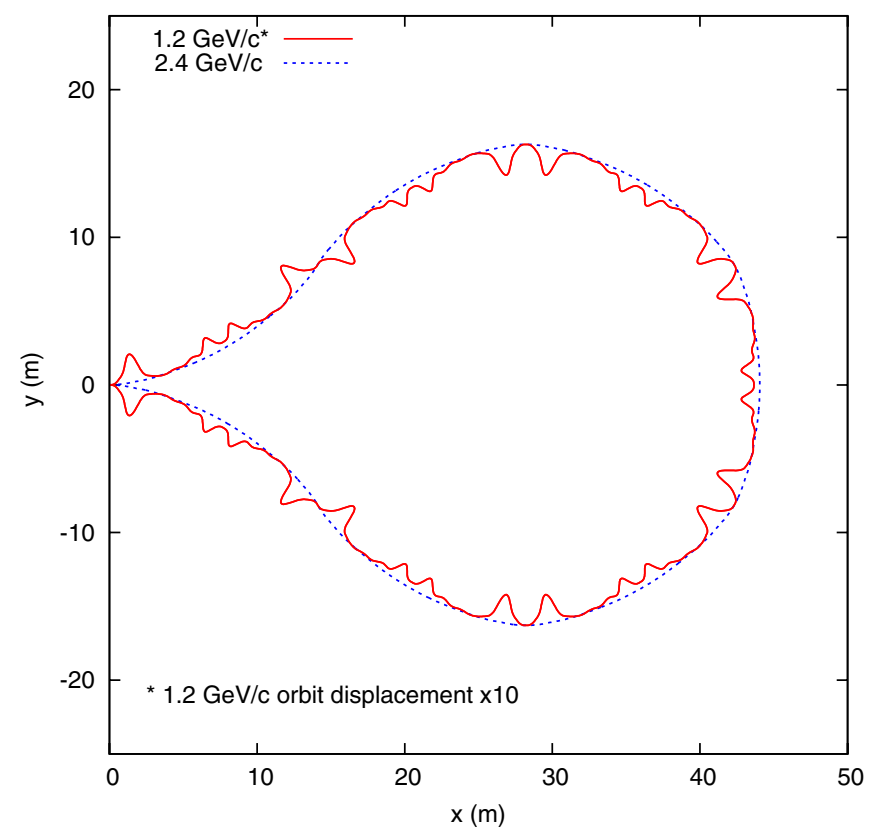

FIG. 6. Geometric layout of the 1.2 and $2.4 \mathrm{GeV} / c$ reference orbits. The displacement of the $1.2 \mathrm{GeV} / c$ orbit is magnified by a factor of 10 .

Note, in particular, the matching points between the superperiods bending in the same and opposite directions. The $2.4 \mathrm{GeV} / c$ optics of a complete arcs can be constructed similarly using the data in Fig. 4.

Figure 6 shows geometric layout of the 1.2 and $2.4 \mathrm{GeV} / c$ reference orbits. The displacement of the $1.2 \mathrm{GeV} / c$ orbit was enhanced by a factor of 10 . Note that because of the varying bending angles, the arc is not perfectly circular. The largest orbit separation of about $19 \mathrm{~cm}$ is determined primarily by the necessity to spread/recombine the differentmomentum orbits at the beginning of the arc. It sets the requirements on the magnet apertures. However, such a large separation occurs only in a small number of magnets while the remaining magnets may have smaller apertures. The maximum orbit deviation is reduced for smaller momentum ratios such as that of the $1.8 / 3.0 \mathrm{GeV} / c$ arc.

The magnet parameters in Table I and the orbit offsets in Figs. 3-5 are specified with respect to a nominalmomentum trajectory, which defines the nominal axis of the magnetic channel. As discussed above, the nominal momentum in our case was set at $2.4 \mathrm{GeV} / c$. In practice, however, the actual magnets do not have to be centered on the channel's nominal axis. In fact, to optimally use the magnets' good-field regions and minimize the required apertures, the center of each physical magnet transversely should be placed approximately halfway between the 1.2 and $2.4 \mathrm{GeV} / c$ orbits. Linearity of the magnetic field allows for a straightforward transformation of the dipole component (the quadrupole component is unchanged) to account for the transverse shift.

\section{TRACKING RESULTS}

To validate our linear optics design, we used the PTC module of MAD-X to track a bunch of 3000 muons through the droplet arc. The tracking results for the 1.2 and $2.4 \mathrm{GeV} / c$ passes are shown in Figs. 7 and 8 respectively. In each case, the initial bunch distribution was Gaussian with no cross correlations between the 6D phase-space coordinates. At each momentum, the horizontal $\varepsilon_{x N}$ and vertical $\varepsilon_{y N}$ normalized rms emittances were both $30 \mathrm{~mm}$ mrad, the rms bunch length $\sigma_{z}$ was $1 \mathrm{~cm}$, and the rms relative momentum spread $\Delta p / p$ was $1 \times 10^{-3}$.

Figures 7 and 8 compare the horizontal (a), vertical (b), and longitudinal (c) phase-space distributions of the initial bunch (shown in blue) to those after the bunch's single pass through the complete droplet arc (shown in red). We intentionally chose the values of the initial transverse and longitudinal emittances at the levels where the bunch was beginning to get deformed at $1.2 \mathrm{GeV} / c$ to probe the limits of the arc's dynamic aperture and momentum acceptance. Note, in particular, in Fig. 7(c) that the longitudinal dynamics starts getting affected by the amplitude path-length dependence, which is a general problem of largetransverse-emittance beams [17]. The particle transmission rates (not including muon decay) were $92.8 \%$ at $1.2 \mathrm{GeV} / c$ and $100 \%$ at $2.4 \mathrm{GeV} / c$.

The results shown in Figs. 7 and 8 indicate that, even without any nonlinear optimization, the arc's dynamic aperture might be adequate for deeply cooled muon beams $[18,19]$. To accommodate a large momentum spread characteristic of muon beams, the arc's longitudinal dynamics
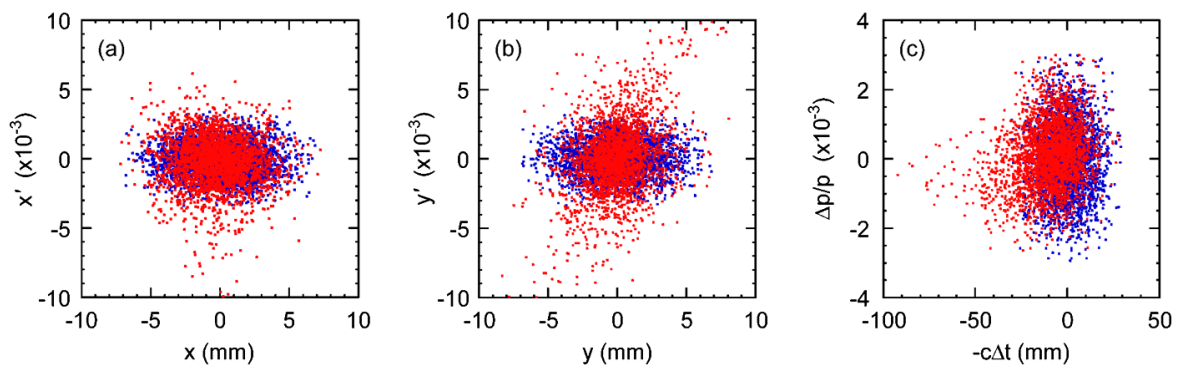

FIG. 7. Horizontal (a), vertical (b), and longitudinal (c) phase-space distributions of a $1.2 \mathrm{GeV} / c$ muon bunch before (blue) and after (red) passing through the droplet arc. 

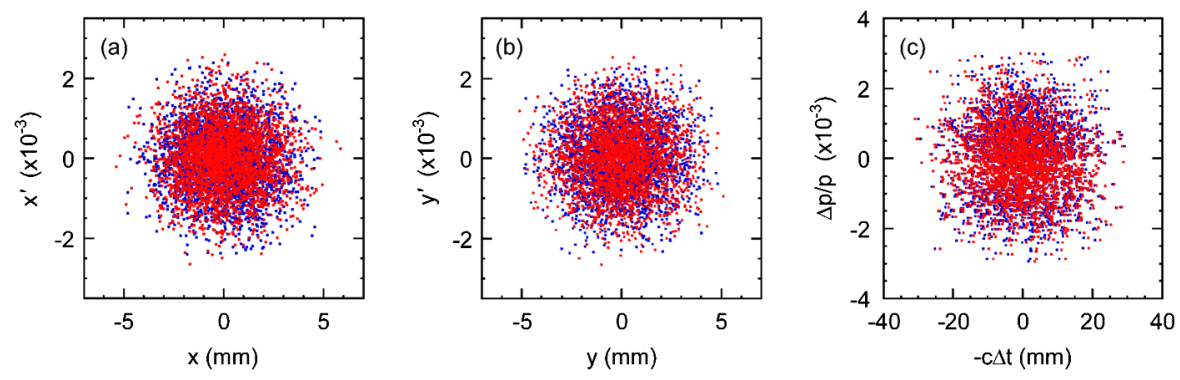

FIG. 8. Horizontal (a), vertical (b), and longitudinal (c) phase-space distributions of a $2.4 \mathrm{GeV} / c$ muon bunch before (blue) and after (red) passing through the droplet arc.

needs to be optimized by compensating chromatic and amplitude-dependent path-length effects and preparing a proper initial bunch state. On the other hand, even without any optimization, the arc properties readily meet the dynamic aperture and momentum acceptance requirements for the typical accelerator beam emittances of most other particle species, such as electrons, protons, deuterons, etc. The tracking in Figs. 7 and 8 was performed for an ideal lattice with no magnet misalignments or magnetic field errors. Studying the sensitivity to such imperfections is not one of the goals of this paper; however, preliminary simulations of these effects seem rather encouraging.

\section{MATCHING TO LINAC AND COMPLETE RLA DESIGN CONSIDERATIONS}

A complete end-to-end RLA design is beyond the scope of this paper. However, as a proof of principle, one can design a corresponding linac with energy gain of $600 \mathrm{MeV}$ per pass, which is matched by design to previously described two-pass arcs for both passes simultaneously. As illustrated in Fig. 9, this multipass linac optics can be accomplished by adjusting the strengths of the linac's fixed-field quadrupoles. The presented solution was accomplished by modifying the so-called bisected linac profile [20], where the quadrupole strengths increase linearly from the linac's center toward the edges. Perhaps the Twiss parameters at the entrance into the arcs can be further optimized from the point of view of better matching to the linac; optimizing the arc and linac optics is an iterative process.

Another critical issue that needs to be considered when developing a complete RLA design is proper synchronization of all passes with the linac's rf. The difference in the times of flight of the different passes in each arc must be either close to zero or close to an integer number of $\mathrm{rf}$ oscillation periods. The time-of-flight difference between the passes arises due to a combination of the differences in their speeds and path lengths.

Since 1.2 and $2.4 \mathrm{GeV} / c$ muons are not ultrarelativistic, there is a non-negligible effect of their speed difference. As it can be seen from Fig. 6 , the $1.2 \mathrm{GeV} / c$ orbit in our design is clearly longer than the $2.4 \mathrm{GeV} / c$ one. Thus, the time-of-flight difference between the two passes must be adjusted to the nearest integer number (including zero) of rf periods. One option is to manipulate the path lengths inside the arc. For instance, one can make $1.2 \mathrm{GeV} / c$ to be the central momentum instead of $2.4 \mathrm{GeV} / c$ in the arc's inner superperiods, which would compensate some of the time-of-flight difference. Another option is to place

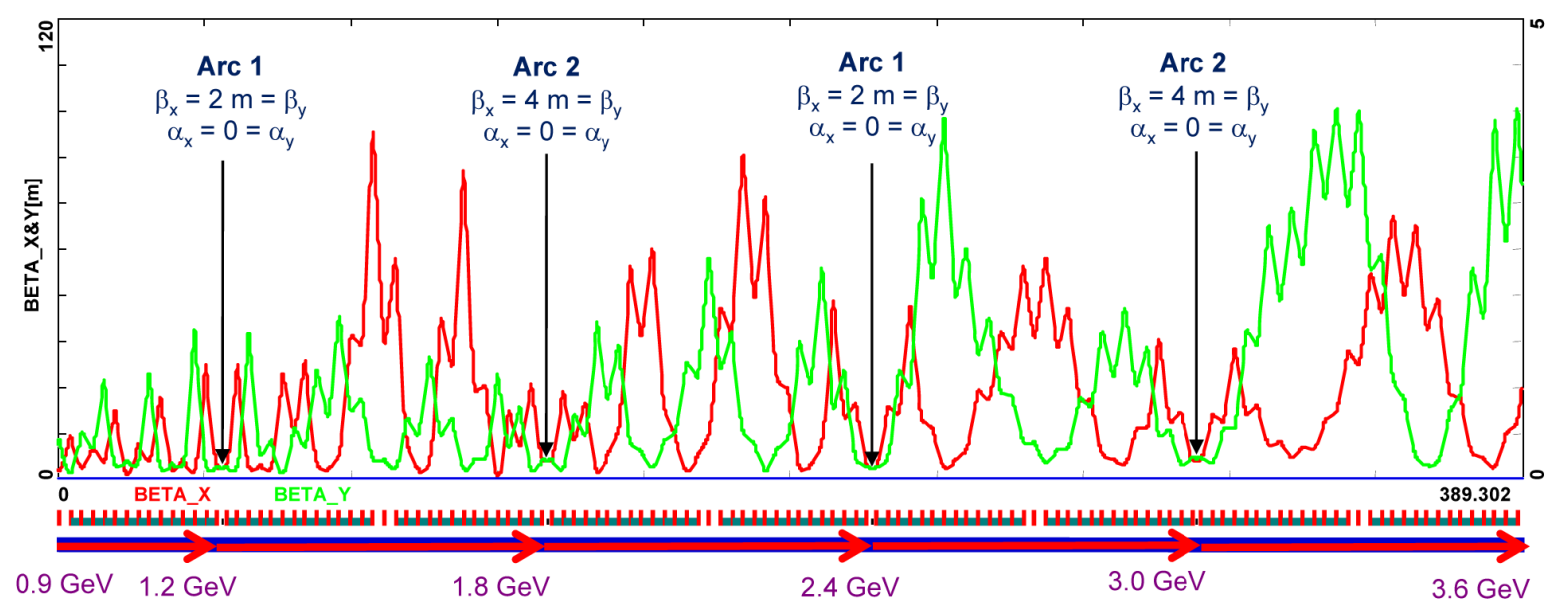

FIG. 9. Linac optics matched to both arcs for all passes simultaneously. The arrows indicate arc locations. 
path-length chicanes [21,22] between the superperiods or in front of the arc. A chicane located in front of the arc would have a cumulative effect on the opposite-direction passes. Combining both options might be the best approach.

\section{CONCLUSIONS}

We developed a concept of an RLA return arc based on linear combined-function magnets, in which two and potentially more consecutive passes with very different energies are transported through the same string of magnets. We demonstrated that, by adjusting the dipole and quadrupole field components of the constituting linear combined-function magnets, the arc can be made achromatic and to have zero initial and final reference orbit offsets for two beam energies. Such an approach combines compactness of the design with the many benefits $[13,14]$ of a conventional linear NS-FFAG scheme.

We applied the concept to design a droplet-shaped return arc for a dogbone muon RLA capable of transporting two beam passes with momenta different by a factor of 2 . We obtained solutions for the reference orbits and linear optics of the two passes. Tracking simulations of the developed design showed that, with appropriate optimization of the longitudinal dynamics, it may be adequate for deeply cooled muon beams and it readily meets the dynamic aperture and momentum acceptance requirements for the typical accelerator beam emittances of most other particle species. There is a straightforward path to a complete RLA design. In that regard, we showed a proof-of-principle transverse matching of the arc to the linac for all passes simultaneously and discussed synchronization of the different passes with the linac's rf.

The proposed multipass arc concept has a number of attractive features. It can increase the number of passes through the linac, thus, leading to a more efficient use of $\mathrm{rf}$ and higher energies available with the same-size accelerator footprint. It eliminates the need for a complicated switch-yard, it reduces the total beam-line length, there is no need to accommodate multiple beam lines in the same tunnel or construct separate tunnels for individual arcs. The design relies solely on fixed magnetic fields, thus, weakening the requirements on magnets and power supplies and greatly simplifying injection and extraction. It allows for a $\mathrm{CW}$ operation. Engineering design and fabrication of linear-field combined-function magnets does not seem to present a challenge [4] even for relatively large apertures. Thus, the multipass approach may benefit many applications in science and industry.

\section{ACKNOWLEDGMENTS}

This manuscript has been authored by Jefferson Science Associates, LLC under Contract No. DE-AC0506OR23177 with the U.S. Department of Energy. This work was supported in part by the U.S. Department of Energy Small Business Technology Transfer Grant No. DE-FG02-08ER86351. We are grateful to A.M. Hutton, R. P. Johnson, G. A. Krafft, and D. Trbojevic for their help and advice.

[1] R.E. Rand, Recirculating Electron Accelerators (Harwood Academic Publishers, New York, 1984).

[2] C. W. Leemann, D. R. Douglas, and G. A. Krafft, Annu. Rev. Nucl. Part. Sci. 51, 413 (2001).

[3] K. B. Beard, R. P. Johnson, S. A. Bogacz, and G. M. Wang, in Proceedings of the 23rd Particle Accelerator Conference, Vancouver, Canada, 2009 (IEEE, Piscataway, NJ, 2009), WE6PFP097, p. 2733.

[4] G. H. Biallas, N. Belcher, D. Douglas, T. Hiatt, and K. Jordan, in Proceedings of the 2007 Particle Accelerator Conference, Albuquerque, New Mexico (IEEE, New York, 2007), MOPAS074, p. 602.

[5] S. A. Bogacz, G. Wang, and R. P. Johnson, in Proceedings of the 23rd Particle Accelerator Conference, Vancouver, Canada, 2009 (Ref. [3]), WE6PFP100, p. 2742.

[6] G. M. Wang, R. P. Johnson, S. A Bogacz, and D. Trbojevic, in Proceedings of the 23rd Particle Accelerator Conference, Vancouver, Canada, 2009 (Ref. [3]), WE6PFP098, p. 2736.

[7] Accelerators for America's Future, DOE Report, June 2010 [http://www.acceleratorsamerica.org/files/Report.pdf].

[8] S.S. Kurennoy, A.J. Jason, and H. Miyadera, in Proceedings of the International Particle Accelerator Conference, Kyoto, Japan (ICR, Kyoto, 2010), WEPE075, p. 3518.

[9] R. W. Hamm and M. E. Hamm, Phys. Today 64, 46 (2011).

[10] CAARI 2012: 22nd International Conference on the Application of Accelerators in Research and Industry, Fort Worth, TX [http://www.caari.com/].

[11] D. Trbojevic, E. D. Courant, and M. Blaskiewicz, Phys. Rev. ST Accel. Beams 8, 050101 (2005).

[12] V.S. Morozov, S.A. Bogacz, and D. Trbojevic, in Proceedings of the International Particle Accelerator Conference, Kyoto, Japan (Ref. [8]), WEPE084, p. 3539.

[13] V.S. Morozov, S. A. Bogacz, Y. R. Roblin, K. B. Beard, and D. Trbojevic, in Proceedings of the 2011 Particle Accelerator Conference, NY, USA (IEEE, New York, 2011), MOP052, p. 196.

[14] V.S. Morozov, S. A. Bogacz, Y.R. Roblin, and K. B. Beard, in Proceedings of the 2nd International Particle Accelerator Conference, San Sebastian, Spain, 2011 (EPS-AG, San Sebastian, Spain, 2011), MOPZ031, p. 868.

[15] E. Forest, F. Schmidt, and E. McIntosh, CERN Report No. CERN-SL-2002-044 (AP) (2002); KEK Report No. 2002-3 (2002) [http://cern.ch/Frank.Schmidt/report/ sl-2002-044.pdf].

[16] http://cern.ch/madx.

[17] J.S. Berg, Nucl. Instrum. Methods Phys. Res., Sect. A 570, 15 (2007).

[18] Ya. S. Derbenev and R.P. Johnson, in Proceedings COOL'05, AIP Conf. Proc. No. 821 (AIP, Melville, NY, 2006), p. 420. 
[19] V.S. Morozov, Ya.S. Derbenev, A. Afanasev, R. P. Johnson, B. Erdelyi, and J. A. Maloney, in Proceedings of the 2nd International Particle Accelerator Conference, San Sebastian, Spain, 2011 (Ref. [14]), WEPZ009, p. 2784.
[20] S. A. Bogacz, in Proceedings NuFact'09, AIP Conf. Proc. No. 1222 (AIP, Melville, NY, 2010), p. 363.

[21] H. A. Enge, Rev. Sci. Instrum. 34, 385 (1963).

[22] H. L. Owen and P. H. Williams, Nucl. Instrum. Methods Phys. Res., Sect. A 662, 12 (2012). 